\title{
A Case Report of Lupus Panniculitis in a Hemodialysis Patient with Lupus Nephritis
}

\author{
Zhi Xiao*, Yanyu Li \\ Department of Nephrology, Binzhou People’s Hospital, Binzhou, China \\ Email: *xz198845@163.com
}

How to cite this paper: Xiao, Z. and Li, Y.Y. (2021) A Case Report of Lupus Panniculitis in a Hemodialysis Patient with Lupus Nephritis. Case Reports in Clinical Medicine, 10, 85-91.

https://doi.org/10.4236/crcm.2021.104010

Received: February 18, 2021

Accepted: March 20, 2021

Published: March 23, 2021

Copyright (c) 2021 by author(s) and Scientific Research Publishing Inc. This work is licensed under the Creative Commons Attribution International License (CC BY 4.0).

http://creativecommons.org/licenses/by/4.0/

(c) (i) Open Access

\begin{abstract}
Background: Systemic lupus erythematosus and lupus nephritis are relatively common autoimmune diseases that can cause damage to multiple systems. Aim: To investigate the lupus activity of patients with lupus nephritis on hemodialysis and the combined occurrence of lupus panniculitis. Case introduction: A patient with lupus nephritis on regular hemodialysis had a symmetrical hard mass under the skin of his abdomen. After surgical resection and pathological examination, she was diagnosed with lupus panniculitis and was treated with glucocorticoids and hydroxychloroquine. After that, no new subcutaneous masses appeared, and the unresectable part of the masses did not increase. Conclusion: 1) Lupus activities in patients with lupus nephritis entering end-stage renal disease still need to be paid attention to. 2) Lupus panniculitis can occur on the skin of the abdomen, and it needs to be differentiated from connective tissue panniculitis, sclerosing panniculitis and other diseases.
\end{abstract}

\section{Keywords}

Lupus Nephritis, Hemodialysis, Lupus Panniculitis

\section{Introduction}

Systemic lupus erythematosus is a systemic autoimmune disease, which is clinically characterized by recurring lupus activities. It is generally believed that lupus activity will decrease after patients with lupus nephritis enter end-stage renal disease and regularly undergo hemodialysis, but there are still extrarenal manifestations of lupus activity in some dialysis patients. This article aims to further explore the clinical diagnosis, treatment and distinguishing characteristics of lupus panniculitis in hemodialysis patients with lupus nephritis, and to improve the early diagnosis rate of the disease. 


\section{Case Data}

A 30-year-old female patient was diagnosed with systemic lupus erythematosus for 9 years. She was admitted to the hospital because of abdominal wall induration for half a year. The patient developed erythema sclerosis on both lower extremities without obvious cause 9 years ago, accompanied by pain, no joint pain, no obvious hair loss, occasional oral ulcers, and was diagnosed with systemic lupus erythematosus and pulmonary hypertension in our hospital. She was treated with drugs such as penisoners, hydroxychloroquine, and cyclophosphamide, and was discharged from the hospital after her condition improved. After that, she continued to be treated with prednisone, hydroxychloroquine, leflunomide, cyclophosphamide and other drugs, and her condition recurred. When her condition worsened, she was treated with high-dose methylprednisolone and immunoglobulin therapy, and her condition improved after treatment. Three years ago, the patient had no obvious cause for edema of both lower extremities. The pathological examination of renal biopsy was performed in the nephrology department of our hospital. The renal pathological results showed lupus nephritis (WHO type IV-Ga/c type). Subsequently, the patient received continuous cyclophosphate, Amide treatment (cumulative $3.8 \mathrm{~g}$ ). During the treatment, the patient's condition repeatedly aggravated and appeared ecchymosis on both lower limbs. One year ago, the patient began to develop symptoms of chest tightness, suffocation, and dyspnea, and was again hospitalized in the intensive care unit and nephrology department of our hospital. After a complete examination, the patient was diagnosed with uremia, combined with heart failure, and was given anti-infection, hemodialysis and other treatments. She was discharged from the hospital after her condition improved, and regular hemodialysis treatment was performed after she was discharged. Six months ago, the patient discovered that there were multiple hard nodules in the abdomen, no pain or special discomfort. The hard nodules under the skin of the abdomen were gradually enlarged without timely diagnosis and treatment. This time she came to our hospital again for systematic diagnosis and treatment. She had a history of hypertension for 2 years. She underwent semi-permanent hemodialysis catheter placement in the right internal jugular vein and an arteriovenous fistula angioplasty in the left forearm 1 year ago. The semi-permanent hemodialysis catheter was removed 5 months ago. Admission examination: T $36.6^{\circ} \mathrm{C}, \mathrm{P} 98$ beats $/ \mathrm{min}$, R 20 beats/min, BP 165/114mmHg. There was no edema in two eyelids, pale eyelid conjunctiva, thick breath sounds in two lungs, no obvious dry and wet rales. The heart rate is 98 beats/min, regular heart rhythm, and with no pathological murmur in the auscultation area of each valve. The abdomen is soft, and multiple nodules can be palpable under the skin on both sides of the abdomen. The texture is hard, granular, non-tender, and the larger one is about $3 \times 2 \mathrm{~cm}$. There was no tenderness, no rebound pain and muscle tension in the abdomen. The liver and spleen were not palpable under the ribs. There was an arteriovenous fistula surgical scar on the left forearm, vascular tremor was palpable, and vas- 
cular murmur was heard on auscultation. No purpura on both lower limbs, no edema on both lower limbs.

Auxiliary examination after admission: echocardiography: left heart enlargement, ventricular septum thickening, aortic valve calcification and a small amount of regurgitation, moderate tricuspid regurgitation, pulmonary hypertension (mild), and left ventricular diastolic function decreased. Abdominal musculoskeletal ultrasound: the mixed echo area is explored between the subcutaneous abdominal external oblique muscles and the fat layer on both sides of the abdomen, the right range is $9.0 \times 3.6 \times 1.8 \mathrm{~cm}$, the left range is $8.7 \times 3.7 \times 1.8$ $\mathrm{cm}$, and the boundary with surrounding tissues is not clear, Which is dominated by a slightly strong echo, accompanied by irregular no echo. Lung CT: ground glass density nodules in the upper lobe of the left lung. Serum immunity: histone positive (+), RO-52 antibody positive (+), anti-U1-nRNP positive (+), anti-nuclear antibody 1:1000 positive. Blood routine: hemoglobin $84 \mathrm{~g} / \mathrm{L}$, erythrocyte sedimentation rate $120 \mathrm{~mm} / \mathrm{h}$, high-sensitivity C-reactive protein 35.10 $\mathrm{mg} / \mathrm{L}$. Serum biochemistry: urea $19.65 \mathrm{mmol} / \mathrm{L}$, creatinine $643 \mu \mathrm{mol} / \mathrm{L}$, uric acid $452 \mu \mathrm{mol} / \mathrm{L}$, total protein $88.56 \mathrm{~g} / \mathrm{L}, \operatorname{IgG} 25.62 \mathrm{~g} / \mathrm{L}, \operatorname{IgA} 4.23 \mathrm{~g} / \mathrm{L}, \operatorname{IgM} 0.15 \mathrm{~g} / \mathrm{L}$, globulin $47.33 \mathrm{~g} / \mathrm{L}$. Abdominal CT: thickened and blurred subcutaneous fat fibers in the abdominal wall (as shown in Figure 1). After the patient was admitted to the hospital, hemodialysis was continued, and the tumor on the right side of the abdominal wall was removed. The pathological results after the resection showed that the submitted tissue (subcutaneously on the abdominal wall) showed changes in panniculitis, visible adipose tissue hyalinosis and membranous necrosis, local calcification, See lymphocyte proliferation with follicle formation, and lymphocyte infiltration of small blood vessel walls (as shown in Figure 2). Combined with clinical data and medical history, it is highly possible to consider lupus panniculitis.

Glucocorticoid and hydroxychloroquine were given during subsequent treatment, and the patient was discharged after stable condition. The patient was followed up after discharge. She continued regular hemodialysis and regularly took glucocorticoids and hydroxychloroquine. No new subcutaneous masses appeared, and the unremoved part of the masses did not increase, and her condition improved.

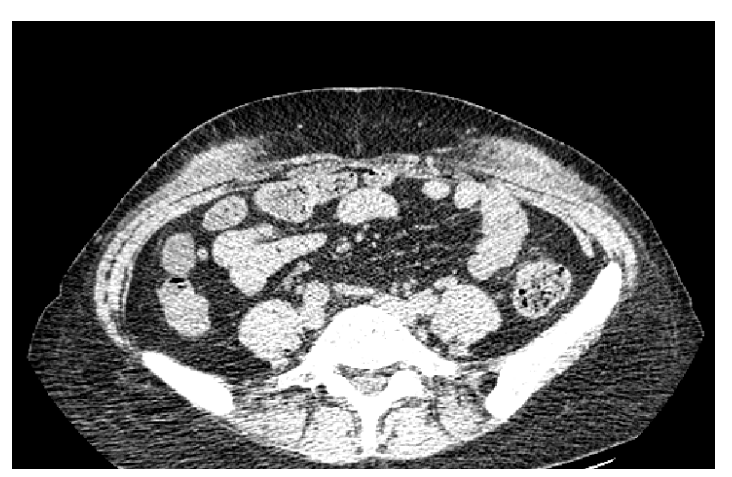

Figure 1. CT manifestations of abdominal wall lupus panniculitis. 


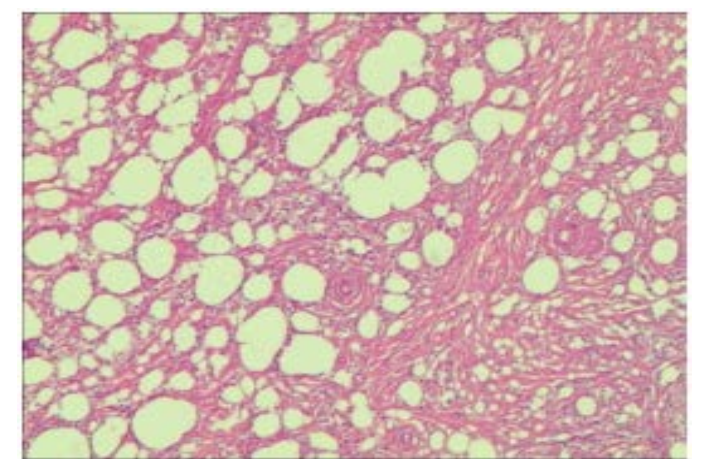

Figure 2. Histopathology of lupus panniculitis in this patient.

\section{Discussion}

Systemic lupus erythematosus is a systemic autoimmune disease that can affect multiple organs. It is clinically characterized by repeated lupus activities, which can affect the skin, muscles and joints, heart, lungs, kidneys, nervous system, blood system, etc. Almost all patients with systemic lupus erythematosus are accompanied by kidney damage [1], and approximately $20 \%$ of patients can develop end-stage renal failure within 10 years after the diagnosis of lupus nephritis and require long-term renal replacement therapy. It has been reported that after lupus nephritis enters the end-stage renal disease stage and undergoes renal replacement therapy, the lupus activity of the organs outside the kidney will be reduced, but the reason is not clear. It may be related to the inhibition of immune cell activity by uremic toxin [2], but there are also studies that have found that patients with lupus nephritis who regularly undergo renal replacement therapy still have lupus activity or new organ lupus activity. The patient reported in this article has been diagnosed with systemic lupus erythematosus for 9 years. She began to enter end-stage renal disease and undergoes regular hemodialysis a year ago. However, combined with the results of the post-admission examination, the patient has anemia, increased erythrocyte sedimentation rate, and immunoglobulin and increased autoantibody titer, considering that the patient still has lupus activity.

Current research shows that for patients with lupus nephritis, uremia and renal replacement therapy can reduce but not completely prevent lupus activity. Studies have shown that with the extension of dialysis time, the positive rates of antinuclear antibodies, anti-double-stranded DNA antibodies, and hypo-C3emia in patients with lupus nephritis are reduced, but the serum immunological indicators of lupus nephritis after end-stage renal failure Abnormality can still be used as an indicator of lupus activity [3]. At the same time, Szeto et al. [4] found that age was associated with lupus nephritis and lupus activity in renal failure. The patient reported in this article is a young female patient. After summarizing her clinical data, we believe that although the patient has undergone regular hemodialysis treatment, there is still extra-renal lupus activity. And we consider that the patient's lupus panniculitis is related to her lupus activity. The lupus ac- 
tivity that enters end-stage renal disease is mainly manifested in the abnormalities of the blood system and serum immunological indicators, and there are few reports about lupus panniculitis. In the subsequent treatment, we gave a small dose of glucocorticoid and hydroxychloroquine to treatment.

Lupus panniculitis, also known as deep-seated lupus erythematosus, is an intermediate type between discoid lupus erythematosus and systemic lupus erythematosus. It is mainly seen in middle-aged women, but it can also occur in a small number of children [5] [6]. It is characterized by relatively hard deep inflammatory nodules under the skin, depression and atrophy of the skin surface, or ulcers and necrosis in the central part, which is single or scattered. Lupus panniculitis usually occurs in the skin of the head, face, upper limbs, and buttocks. Among the patients reported in this article, lupus panniculitis occurs under the skin of the abdomen, which is relatively rare. The pathogenesis of lupus panniculitis is currently unclear, and it is generally believed to be related to multiple factors such as genetics, drugs, viruses, physical factors, and sex hormones. The pathological manifestations of lupus panniculitis are more complicated, and its pathological changes may be related to the course of the disease. Requena et al. [7] pointed out that lobular panniculitis is the basic pathological change of lupus panniculitis. Peters et al. [8] studied the necessary criteria for the pathological diagnosis of lupus panniculitis: 1) lymphocytes showed follicular infiltration; 2) fatty necrosis; 3) lobular panniculitis or septal panniculitis; 4) calcification. Secondary criteria: 1) The epidermis is manifested by discoid lupus erythematosus; 2) lymphocytic or vascular vasculitis; 3) zonal hyaline degeneration under the epidermis; 4) mucin deposition; 5) plasma cell or eosinophil infiltration; 6) tissue. The cells can show granulomatous changes. In the histopathology of this patient reported in this article, we can also see adipose tissue hyalinosis and membranous necrosis, local calcification, lymphocyte proliferation, follicular formation, and lymphocytic infiltration of the vessel wall. These pathological changes are consistent with related reports in the literature.

Lupus panniculitis needs to be differentiated from connective tissue panniculitis, sclerosing panniculitis, nodular febrile non-suppurative panniculitis, hard erythema and other diseases in histopathology, and special attention should be paid to subcutaneous Differentiation of panniculitis-like T-cell lymphoma [9]. Subcutaneous panniculitis-like T-cell lymphoma is a cytotoxic T-cell lymphoma derived from $\alpha \beta$. It is characterized by panniculitis-like changes in infiltration of subcutaneous fat. The early stage and lupus panniculitis are both panniculitis-like damage. In histopathological examination, the lesions of subcutaneous panniculitis-like T-cell lymphoma hardly involve the dermis, and the infiltrating atypical lymphocytes are generally confined to the subcutaneous fat layer. The characteristic manifestation in pathological examination is the formation of flowers around the fat cell space. Ring structure [10]. For the treatment of lupus panniculitis, some studies believe that hydroxychloroquine sulfate has a better response to the treatment of lupus panniculitis and can be used as the first-line 
medication for the treatment of lupus panniculitis. However, for patients with ANA positive or severe skin rash, it is still requires combined glucocorticoid therapy [11]. Most patients need to take the medicine for a long time and reduce the dose slowly. In addition, hydroxychloroquine sulfate combined with azathioprine, cyclosporine A, mycophenolate mofetil and other immunosuppressive agents also have broad prospects in the treatment of lupus panniculitis [12].

\section{Conclusion}

Lupus panniculitis can occur on the skin of the abdomen, and it needs to be differentiated from connective tissue panniculitis, sclerosing panniculitis and other diseases. Most patients with lupus nephritis have reduced lupus activity after entering end-stage renal disease, but through this case, we realized that some patients still have lupus activity although they have undergone regular hemodialysis, and such patients still need to continue treatment for lupus.

\section{Conflicts of Interest}

The authors declare no conflicts of interest regarding the publication of this paper.

\section{References}

[1] Ye, R.G. and Liu, G.X. (1997) Nephrology. People's Medical Publishing House, Beijing, 85-88.

[2] Coplon, N.S, Diskin, C.J., Jeffrey, P., et al. (1983) The Long-Term Clinical Course of Systemic Lupus Erythematosus in End-Stage Renal Disease. New England Journal of Medicine, 308, 186-190. https://doi.org/10.1056/NEJM198301273080403

[3] Wang, W., Xia, Y.L., Wei, T., et al. (2003) Clinical and Immunological Analysis of Extrarenal Lupus Activity in End-Stage Lupus Nephritis. Journal of Lanzhou Medical College, 29, 31-33.

[4] Szeto, C.C., Li, P.K., Wong, Y.H., et al. (1998) Factors Associated with Active Systemic Lupus Erythematosus after Endstage Renal Disease. Journal of Rheumatology, $25,1520-1525$.

[5] Moreno-Suárez, F. and Pulpillo-Ruiz, Á. (2013) Rituximab for the Treatment of Lupus Erythematosus Panniculitis. Dermatologic Therapy, 26, 415-418. https://doi.org/10.1111/dth.12014

[6] Lei, H., Ma, G.E and Liu, Z. (2016) Evaluation of Repairing Facial Depression Deformities Secondary to Lupus Erythematosus Panniculitis with Autologous Fat Grafting. Journal of Craniofacial Surgery, 27, 1765-1769. https://doi.org/10.1097/SCS.0000000000003073

[7] Requena, L. (2007) Normal Subcutaneous Fat, Necrosis of Adipocytes and Classification of the Panniculitides. Seminars in Cutaneous Medicine \& Surgery, 26, 66-70. https://doi.org/10.1016/j.sder.2007.02.001

[8] Peters, M.S. and Su, W.P. (1991) Eosinophils in Lupus Panniculitis and Morphea Profunda. Journal of Cutaneous Pathology, 18, 189-192. https://doi.org/10.1111/j.1600-0560.1991.tb00151.x

[9] Massone, C., Kodama, K., Salmhofer, W., et al. (2010) Lupus Erythematosus Panniculitis (Lupus Profundus): Clinical, Histopathological, and Molecular Analysis of 
Nine Cases. Journal of Cutaneous Pathology, 32, 396-404.

https://doi.org/10.1111/j.0303-6987.2005.00351.x

[10] Zhang, S.H., Shao, H.L., Yue, B., et al. (2020) Clinicopathological Analysis of Subcutaneous Panniculitis-Like T-Cell Lymphoma. Journal of Diagnostic Pathology, 27, 689-692.

[11] Wang, T., Li, X., Liu, Y.H., et al. (2011) Clinical and Pathological Analysis of 22 Cases of Lupus Panniculitis. Journal of Chinese Academy of Medical Sciences, 33, 525-528.

[12] Santo, J.E., Gomes, M.F., Gomes, M.J., et al. (2010) Intravenous Immunoglobulin in Lupus Panniculitis. Clinical Reviews in Allergy \& Immunology, 38, 307-318.

https://doi.org/10.1007/s12016-009-8162-x 